Journal of Engineering and Applied Sciences 14 (Special Issue 1): 3950-3953, 2019

ISSN: 1816-949X

(C) Medwell Journals, 2019

\title{
Component Measurement for Scale and Adaptability of Self-Adaptive Systems
}

\author{
${ }^{1}$ Jung Hyun An and ${ }^{2}$ Young B. Park \\ ${ }^{1}$ Department of Computer Engineering, Graduate School, \\ ${ }^{2}$ Department of Software Science, Dankook University, Yongin, Korea
}

\begin{abstract}
With the development of artificial intelligence technology and the expansion of the IoT concept, the problem of self-adaptability of the system which can respond to a given situation without system intervention is becoming important. Many self-adaptive systems implement self-adaptation to adaptation objects through implicitly or explicitly defined feedback loop processes. Therefore, in the feedback loop process, defining the monitoring elements for the adaptation object, the states of the respective elements and the corresponding plans for the identified states is directly related to realizing the system's self-adaptability. Existing self-adaptive systems are designed to implement self-adaptability for a specific area. Many studies have proposed a method and framework for implementing a general adaptive self-adaptive system but the complexity that the system must deal with increases as the number of adaptive elements increases. Due to the characteristics of the self-adaptive system it is a very difficult problem to confirm the validity of all adaptation results. In this study, we propose a method to calculate the possible combinations of states and the corresponding plans in the feedback loop of the system to verify the validity of the adaptation results in the self-adaptation process. This methodology defines the adaptation object of a self-adaptive system as a set of elements that can have a finite number of states and then calculates the number of state combinations that can be generated by the elements in each feedback loop and the corresponding plan. The computed result can serve as metadata that can implement a self-adaptive system because it includes complexity, size and operational criteria of the self-adaptive system. It can also be used to determine the suitability of the implemented adaptation results of the adaptive system. We describe the methodology for defining the adaptation process and the membership of the self-adaptive system based on the feedback loop according to the methodology and for calculating the number of cases of state combinations and corresponding plans. In this study, we propose an adaptation system based on the proposed method and compare the result of the proposed method with that of the adaptive system.
\end{abstract}

Key words: Self-adaptive system, feedback loop, component measurement, autonomous system internet of things, framework, adaptation, elements

\section{INTRODUCTION}

The trend of technological advancement represented by $\mathrm{AI}$ intelligent things and conversational platforms is to understand how to automate the tasks that people have had to do before. Adaptability, realized through feedback loops is a key requirement to deal with uncertain operating conditions in CPS (Bordel et al., 2017). The self-adaptation of the system is performed automatically by modifying the attributes or entities of the system according to the change of the target environment (Salehie and Tahvildari, 2009). These self-adaptations are self-optimization to achieve optimization of the system in response to environmental changes, self-defense for dynamic response to external threats, self-adaptation such as self-organization, self-recovery (Muller-Schloer et al., 2011). The self-adaptive system recognizes itself and the situation of adaptive environment and implements adaptability through adaptive logic (Brun et al., 2009). The feedback loop is an approach that classifies the system's adaptive logic and processes into environmental monitoring, analysis, response planning and execution. Many SASs have implicitly or explicitly introduced feedback loops into the system.

MAPE-K (Monitoring, Analyze, Plan, Execute and Knowledge) (Iglesia and Weyns, 2015) is the most explicit model of the role of the feedback loop. Each member is explicitly separated and implemented in modules within the system. Knowledge stores and processes information for the environment and the system itself and members of the feedback loop work with reference to common knowledge. Damien's study Borgetto et al. (2015) has shown that the implementation of a self-adaptive system based on the MAPE-K Model in a distributed cloud

Corresponding Author: Young B. Park, Department of Software Science, Dankook University, Yongin, Korea 
environment can dynamically adjust the energy consumption of the environment members to the changing environment.

The self-adaptive system for a small-scale adaptation environment can handle the identifiable state and the problem state for the environment to be considered within a human perception range but a large-scale self-adaptation considering all the states and response plans in the system is a very complex problem. From the above problems, another problem arises that must be handled in implementing a general-purpose adaptive system. In this study, to measure the size of the self-adaptive process in the system using the feedback loop-based self-adaptive process and to clearly identify the result of each adaptive action in the process and a method of calculating the number in the self-adaptive system, the target environment is defined as a set of elements that can have multiple states and the feedback loop process identifies the states in the elements and plans the countermeasures. The advantage of designing and implementing a self-adaptive system using this method is as.

When designing and implementing a self-adaptive system it is possible to predict the complexity that exponentially increases as the elements of the target environment are added and predict the self-adaptive process that occurs in each feedback loop process.
By verifying that the implemented state combinations and corresponding plans are used within the adaptation system it is possible to clearly see if the adaptive behavior of the person is valid for the problem situation. As a method of confirming the adaptation result, the data of the feedback loop is stored and the system log is checked against the stored data. By formalizing the role, condition and results of the feedback loop within the self-adaptive system it is possible to reduce the design and implementation resources for the more general case and the larger self-adaptive system.

\section{MATERIALS AND METHODS}

A possible apdative component in the self-adaptive system: The calculated state set is defined as a set of valued states that require a corresponding plan in the self-adaptation aspect. This study calculates the number of cases of the corresponding plans for each of these sets of states. The response scheme in the proposed method is defined as a solution to whether the feedback action A is to be delivered during the constant feedback loop cycle $\mathrm{T}$ for the members $\mathrm{P}$ belonging to the environment. Thus, establishing a response plan for a set of problematic states can be summarized as a selection problem for the members of the adaptive environment. Figure 1 shows the process of creating a response plan for a set of problem states.

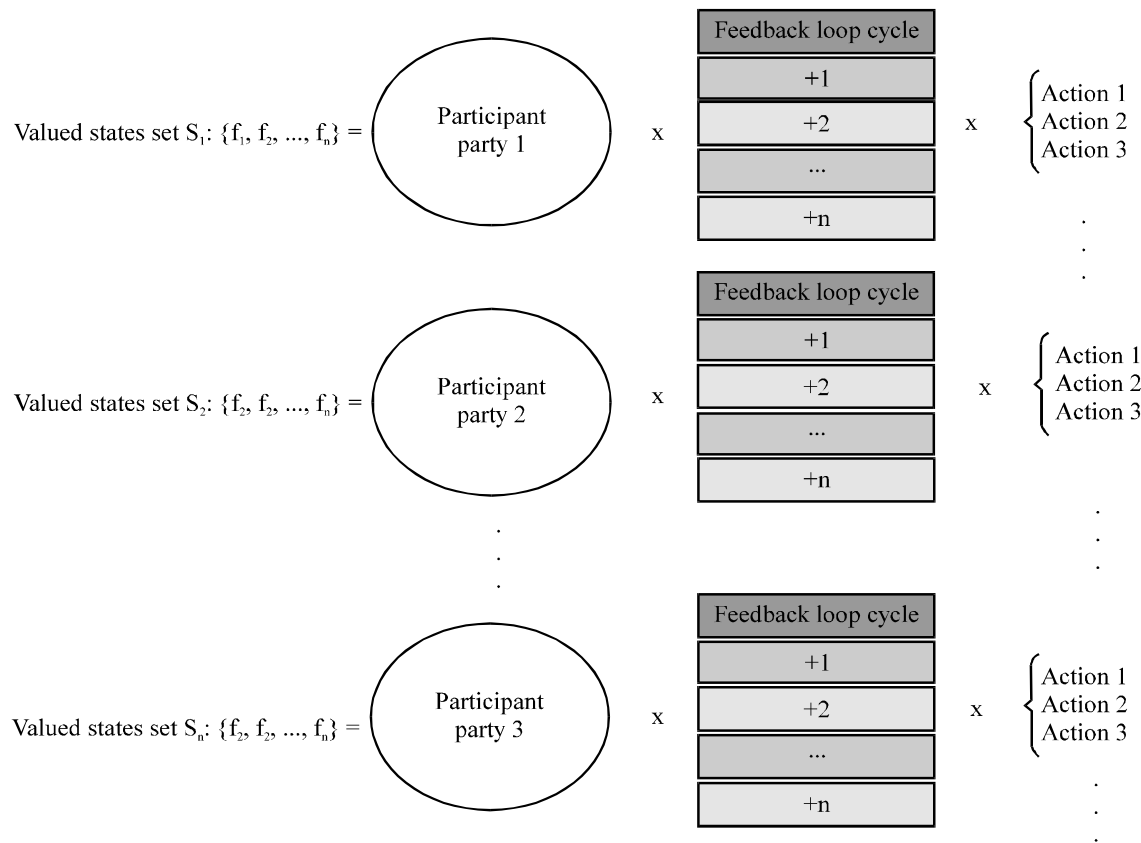

Fig. 1: The process of creating a response plan for a set of problem states 
For the set of problem states, select as many as $r$ members to apply to problem solving in all members $p$. Thereafter, the number of corresponding plans that can be generated by a product of a cycle that can be established and the number of actions for the selected member is calculated. Equation 1 relates to the number calculation in the case of a correspondence plan that can be established:

$$
\text { Response plan }=\sum_{\mathrm{r}=1}^{\mathrm{p}} \mathrm{pCr}^{*} \mathrm{~T}^{*} \mathrm{~A}
$$

\section{RESULTS AND DISCUSSION}

Implementation of a solar panel self-adaptive system and verification of feedback loop using a method: In this research, we introduce the formulas calculated in section 2 into the self-adaptive system for the actual solar panel environment and compare the results obtained in the feedback loop of the system to verify that the system has acted according to the actual calculated formula. The adaptive environment of the self-adaptive system is a power grid environment through an energy storage station that measures the total solar heat generation and manages its energy using inverter sensors on multiple solar power generation panels. The system analyses power generation by storage energy, climate or panel angle and adjusts the power maintenance plan of the grid to match the external power consumption. Figure 2 and Table 1 shows the members of the solar energy grid.

The total number of plans calculated using the number of members in the actual implemented system, the maximum cycle that can be fed back by the system and the type of action is 720 . Finally, Eq. 5 shows that the total number of possible states in the adaptation system of the solar panel developer and the corresponding number of corresponding planning solutions are 36,000 . This figure represents a solution to the problem situation and situation that can occur within the self-adaptation system. The actual self-adaptation system was run and the total environmental analysis performed in the entire feedback

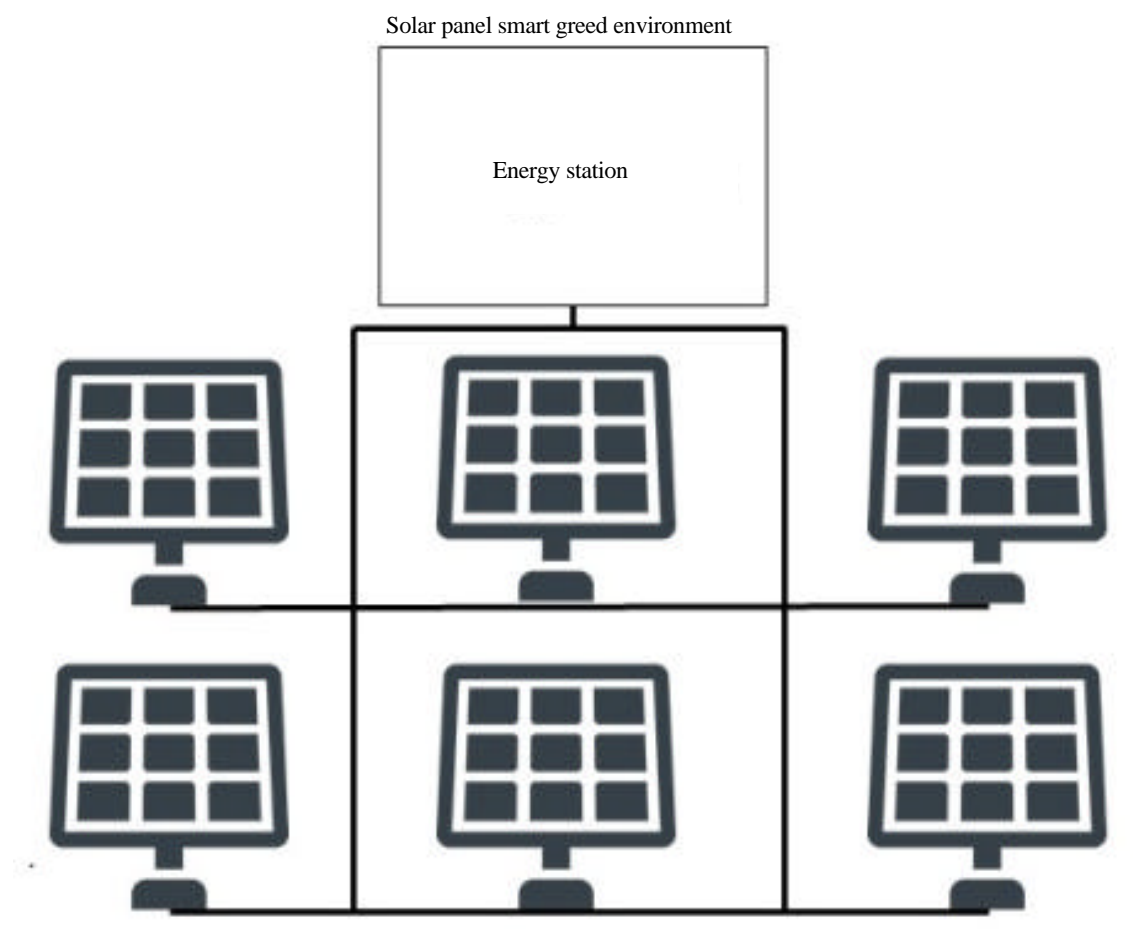

Fig. 2: Metamodel for solar panel smart greed environment

Table 1: Define factor and state of solar panel smart greed environment

\begin{tabular}{|c|c|c|c|c|c|c|}
\hline Factors & $\begin{array}{l}\text { Power } \\
\text { generation }(\mathrm{kW})\end{array}$ & $\begin{array}{l}\text { Panel } \\
\text { temperature }\left({ }^{\circ} \mathrm{C}\right)\end{array}$ & $\begin{array}{l}\text { Energy } \\
\text { reserve }(\mathrm{kW})\end{array}$ & $\begin{array}{l}\text { Solar } \\
\text { radiation }(\mathrm{mm})\end{array}$ & $\begin{array}{l}\text { Power } \\
\text { efficiency (\%) }\end{array}$ & Time (h) \\
\hline 1 & $0 \sim 0.45$ & $-20 \sim 9.9$ & Low & $-198.5 \sim 50$ & $0 \sim 79.92$ & $20 \sim 10$ \\
\hline 2 & $0.5 \sim 1.45$ & $10 \sim 19.9$ & Normal & $-49.9 \sim-10$ & $80 \sim 99.98$ & $11 \sim 14$ \\
\hline 3 & $1.5 \sim 2.45$ & $20 \sim 29.9$ & Over & $-9.9 \sim 9.9$ & $100 \sim 109$ & $15 \sim 20$ \\
\hline 4 & $2.5 \sim 3.45$ & $30 \sim 39.9$ & - & $10 \sim 99.9$ & $110 \sim 129$ & - \\
\hline 5 & $3.5 \sim 5$ & $40 \sim 63.5$ & - & $100 \sim 518.1$ & $130 \sim 390$ & - \\
\hline
\end{tabular}


Table 2: Number of operations of each feedback loop process Feedback loop component name

Monitoring Number of process

Analyzing

Planning

12122

11027

loop cycle and the number of operations of the corresponding planning operation were measured. In this way, we measure how adaptive logic of the self-adaptive system guarantees adaptability to changes in the target environment. Table 2 shows the frequency of analysis and response planning in relation to the overall feedback loop cycle.

\section{CONCLUSION}

In this study, a self-adaptive system implemented using the proposed methodology is described as an example and possible combinations of states and corresponding plans are calculated through a methodology. In addition, we show that the proposed method can be used to design and implement a self-adaptive system through the methodology.

\section{RECOMMENDATIONS}

In the future research, we propose a method to calculate the number of context states and corresponding strategies in a comprehensive IT ecosystem environment that covers multiple feedback loops rather than a single feedback loop. We will study the method of objectively confirming the criterion of whether we can judge whether we are not subjectively by machine learning.

\section{ACKNOWELDGEMENTS}

This research was supported by the MISP (Ministry of Science, ICT and Future Planning), Korea, under the SW master's course of a hiring contract program (H0116-16-1015) supervised by the IITP (Institute for Information and Communications Technology Promotion). This research was supported by the Leading Human Resource Training Program of Regional Neo industry through the National Research Foundation of Korea (NRF) funded by the Ministry of Science and ICT (No.NRF-2016H1D5A1909989).

\section{REFERENCES}

Bordel, B., R. Alcarria, T. Robles and D. Martin, 2017. Cyber-physical systems: Extending pervasive sensing from control theory to the internet of things. Pervasive Mob. Comput., 40: 156-184.

Borgetto, D., M. Maurer, G. Da-Costa, J.M. Pierson and I. Brandic, 2012. Energy-efficient and sla-aware management of IAAS clouds. Proceedings of the 3rd International Conference on Future Energy Systems: Where Energy, Computing and Communication Meet (e-Energy '12), May 09-11, 2012, ACM, Madrid, Spain, Europe, ISBN:978-14503-1055-0, pp: 1-10.

Brun, Y., G.D.M. Serugendo, C. Gacek, H. Giese and H. Kienle et al., 2009. Engineering Self-Adaptive Systems through Feedback Loops. In: Software Engineering for Self-Adaptive Systems, Cheng, B.H., R. de-Lemos, H. Giese, P. Inverardi and J. Magee (Eds.). Springer, Berlin, Heidelberg, Germany, ISBN:978-3-642-02160-2, pp: 48-70.

Iglesia, D.G.D.L. and D. Weyns, 2015. MAPE-K formal templates to rigorously design behaviors for selfadaptive systems. ACM. Trans. Auton. Adapt. Syst., 10: $1-31$.

Muller-Schloer, C., H. Schmeck and T. Ungerer, 2011. Organic Computing-A Paradigm Shift for Complex Systems. Springer, Berlin, Germany, ISBN:978-3-03480130-0, Pages: 627.

Salehie, M. and L. Tahvildari, 2009. Self-adaptive software: Landscape and research challenges. ACM. Trans. Auton. Adapt. Syst., 4: 1-42 